\title{
ORIGINAL
}

\section{DISFUNCIÓN ERÉCTIL EN POBLACIÓN GERIÁTRICA MASCULINA CON HIPERTENSIÓN ARTERIAL EN TEGUCIGALPA HONDURAS}

\author{
ERECTILE DYSFUNCTION IN MALE GERIATRIC POPULATION WITH ARTE- \\ RIAL HYPERTENSION IN TEGUCIGALPA HONDURAS \\ María José Folgar Contreras ${ }^{1}$,Milagromaria Ramos Rodriguez², Keily Sarahi Pastrana Gonzales ${ }^{3}$.
}

1,2,3 Doctora en Medicina y $\mathrm{Ci}$ rugía. Universidad Católica de Honduras.

Dirección: Colonia las Colinas, bloque RR, 4ta casa después del Liceo Franco Hondureño, Tegucigalpa, Honduras.

Correspondencia a: María José Folgar Contreras folgarmj@gmail.com Telf. y celular: +504 98865105

Palabras clave: Disfunción eréctil, Hipertensión, Adulto mayor.

Keywords: Erectile dysfunction, Hypertension, Elderly.

Procedencia y arbitraje: no comisionado, sometido a arbitraje externo.

Recibido para publicación: 20 mayo 2017 Aceptado para publicación 14 julio 2018

Citar como: Rev Cient Cienc Med 2018;21(1):60-67

\section{RESUMEN}

Introducción:Actualmente se reconoce a la Disfunción Eréctil como una enfermedad multifactorial en la que coexisten causas de tipo psicológicas, neurológicas, endocrinológicas, vasculares, traumáticas, estilos de vida, medio ambiente e iatrogénicas. Si bien es cierto que es una enfermedad benigna, afecta tanto la salud física como psicosocial, resultando en la afectación de la calidad de vida de quienes la sufren. Objetivo: Determinar la prevalencia de disfunción eréctil en pacientes masculinos hipertensos que asisten a la consulta externa del Centro de Atención Integral del Adulto Mayor. Metodología: Se realizó un estudio descriptivo transversal, tomando una muestra de 210 individuos de un universo de 465 , obteniendo datos a partir de la aplicación de dos encuestas a los pacientes masculinos e hipertensos en los meses de octubre y noviembre del año 2013. Las encuestas incluían preguntas de carácter general y el Indice Internacional de Función Eréctil (IIEF-5). Resultados: En la muestra se encontró que la prevalencia de disfunción eréctil en los pacientes masculinos hipertensos que asisten a la consulta externa del Centro de Atención Integral del Adulto Mayor fue de 58\%. Conclusiones: En nuestra población geriátrica masculina e hipertensa que asiste a la consulta externa del Centro de Atención Integral del Adulto Mayor hay una prevalencia alta de disfunción eréctil, presentándose con mayor frecuencia en un rango de edad avanzada debido a causa de enfermedades concomitantes.

\section{ABSTRACT}

Introduction: Erectile Dysfunction is currently recognized as a multifactorial disease in which psychological, neurological, endocrine, vascular, traumatic, life-style, environmental and iatrogenic causes coexist. Although it is a benign disease, it affects both physical and psychosocial health, resulting in the affectation of the quality of life of those who suffer it. Objective:To determine the prevalence of erectile dysfunction in hypertensive male patients attending the outpatient clinic of the "Centro de Atención Integral del Adulto Mayor". Methodology: A cross-sectional descriptive study was carried out, taking a sample of 210 individuals from a universe of 465 , obtaining data from the application of two surveys to male and hypertensive patients in the months of October and November of the year 2013. Surveys Included general questions and the International Erectile Function Index (IIEF-5). Results:The sample showed that the prevalence of erectile dysfunction in hypertensive male patients attending the outpatient clinic of the Centro de Atención Integral del Adulto Mayor was $58 \%$. Conclusions: In our male and hypertensive geriatric population attending the outpatient clinic of the Centro de Atencion Integral del Adulto Mayor, there is a high prevalence of erectile dysfunction, presenting more frequently in a range of advanced age due to concomitant diseases.

\section{INTRODUCCIÓN}

a Disfunción eréctil se define como la incapacidad consistente (durable, estable) de lograr o manteLner una erección peneana suficiente como para permitir una relación sexual satisfactoria'. A pesar de que es considerada una enfermedad benigna, tiene alta repercusión en la calidad de vida de la persona que la padece, así como de su pareja, ya que afecta al individuo desde lo físico a lo emocional². 
En el año de 1573 Varilo explicó la erección como producto de un mecanismo de restricción venosa ${ }^{3}$, pero fue a partir de los conceptos de Freud que se le consideró de origen psicológico'. En la actualidad se conocen tres mecanismos básicos que pueden ocasionar disfunción eréctil: incapacidad para iniciar la erección (causa psicógena, endocrina o neurógena), insuficiencia de llenado (arteriógena) y la incapacidad para almacenar un volumen de sangre suficiente en la red lagunar (disfunción veno-oclusiva) ${ }^{4}$.

Se reconoce a la disfunción eréctil como una enfermedad multifactorial en la que coexisten causas de tipo psíquicas, neurógenas, endocrinas; enfermedades concomitantes como diabetes, vasculares, traumáticas; causas relacionadas con fármacos, estilos de vida e incluso se han descrito causas iatrogénicas ${ }^{2}$. La disfunción eréctil y la hipertensión arterial comparten los mismos factores de riesgo (sedenterismo, obesidad, alcohol y tabaquismo), los cuales están a su vez asociados con la disfunción endotelial.

Se ha encontrado una alta prevalencia en pacientes con disfunción eréctil e hipertensión arterial, que puede deberse al daño endotelial en la pared arterial de los cuerpos cavernosos y por el efecto que ciertos fármacos antihipertensivos producen en la erección, siendo los que más afectan betabloqueantes, hidralazina, metildopa y diuréticos ${ }^{6}$. Siendo la hipertensión arterial una enfermedad endotelial crónica muy frecuente, poco controlada y que coexiste con la disfunción eréctil' ${ }^{1}$, es de gran utilidad conocer la frecuencia de la relación entre hipertensión arterial y disfunción eréctil en la población geriátrica.

En este estudio se determinó la prevalencia de disfunción eréctil en sus diferentes grados en los pacientes masculinos hipertensos que asistieron a la consulta externa del Centro de Atención Integral del Adulto Mayor, evidenciando otras enfermedades que coexisten con la disfunción eréctil; la cual tradicionalmente se ha catalogado como un fenómeno resultado de la edad. Si bien es cierto, es concomitante de algunos cambios fisiológicos y psíquicos de la senectud, hoy es considerada una enfermedad 7 . Por esta razón se pretende a través de este, evidenciar la necesidad de manejar integralmente la enfermedad y considerando el aumento de los casos de esta, sugerir crear medidas de prevención.

\section{MATERIALES Y MÉTODOS}

Se realizó un estudio descriptivo transversal, tomando una muestra de 210 hombres de un universo de 465 pacientes masculinos e hipertensos que acudieron al Centro de Atención Integral del Adulto Mayor (CAIAM) en Tegucigalpa, Honduras, en los meses de octubre y noviembre del año 2013. Se tomó como criterios de inclusión a los pacientes del sexo masculino con hipertensión diagnosticada y tratada en el Centro de Atención Integral del Adulto Mayor (CAIAM), con edad entre 60 y 90 años; y consentimiento informado firmado. Se excluyeron hombres sin hipertensión arterial. Los datos se obtuvieron a través de la aplicación de dos instrumentos; luego de haber obtenido el consentimiento informado firmado de cada paciente. La primera fue una encuesta con datos de carácter general y la segunda el IIEF-5 que considera a los pacientes en base a un puntaje de 22-25 sin función eréctil; 17-21 con grado leve, 12-16 con grado leve a moderado, 8-11 con grado moderado, y 5-7 con grado severo. Para el análisis los datos, estos se introdujeron y almacenaron en el programa estadístico Epi-info ${ }^{\circledR}$ versión 3.01. Con ellos, se realizaron tablas y gráficas en Microsoft Excel for $\mathrm{Mac}^{\circledast} 2011$, versión 14.1.0. Se calculó el nivel de confianza de $95 \%$ y con un margen de error del $5 \%$.

\section{RESULTADOS}

Se aplicaron dos encuestas a una muestra de 210 hombres hipertensos, siendo todas llenadas adecuadamente. De estos 210 encuestados, 58\%(122) resultaron con algún grado de disfunción eréctil (Ver grafica 1). La puntación obtenida en el cuestionario IIEF-5 ayudo a obtener la gravedad de la disfunción eréctil (Ver tabla 1); se encontró que, de los 122 hombres con disfunción eréctil, la mayoría presento disfunción eréctil en grado moderado $21 \%(44)$, seguido de disfunción eréctil en grado leve 19\%(40), 13\%(27) grado severo y un 5\%(11) grado moderado a severo (Ver gráfica 2). Se demostró que, en esos 122 hombres con disfunción eréctil, el riesgo de disfunción eréctil fue mayor a partir de los 70 años 43\%(91) (Ver tabla 2). Dentro de esta población de encuestados se estudiaron más hombres de 70 a 80 años 45\%(94).

En los 122 pacientes hipertensos estudiados que presentaron disfunción eréctil, se encontraron otras enfermedades concomitantes. Las enfermedades que más resultaron prevalentes fueron la diabetes mellitus en un $23 \%(48)$ y la enfermedad cardiovascular 19\%(40); sin embargo, un 16\%(34) presentó otras enfermedades aparte de la hipertensión, entre las que están hipercolesterolemia, obesidad y depresión. (Ver gráfica 3)

Gráfica $\quad 1$ 


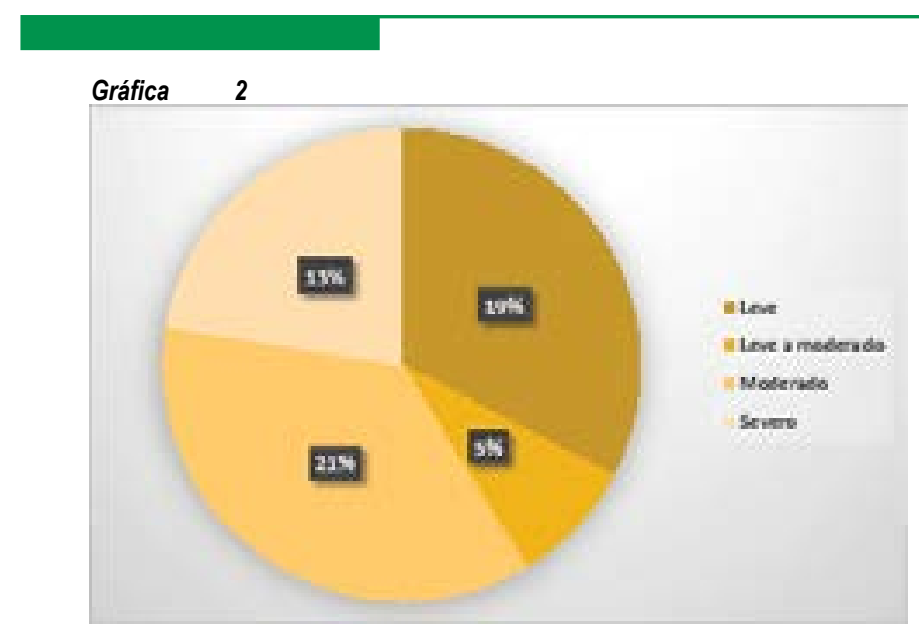

Tabla 1. Grados de disfunción eréctil.

\begin{tabular}{|ll|}
\hline GRADO & Puntaje \\
\hline Sin disfunción eréctil & $22-25$ \\
\hline Grado leve & $17-21$ \\
\hline Grado leve a moderado & $12-16$ \\
\hline Grado moderado & $8-11$ \\
\hline Grado severo & $5-7$ \\
\hline
\end{tabular}

Fuente: http://www.croesoffice.org/Portals/0/Short_IIEF.pdf

\section{DISCUSIÓN}

La prevalencia de la disfunción eréctil varía entre países, regiones y ciudades, se encuentra entre el 10 y el $52 \%$ en hombres entre 40 y 70 años, con una incidencia en países occidentales de 25-30 nuevos casos por cada 1000 habitantes al año ${ }^{8}$. En nuestro estudio realizado en el CAIAM en el año 2013, se logró establecer la prevalencia de la disfunción eréctil en el paciente hipertenso, obteniendo resultados comparables con estadísticas nacionales e internacionales.

Un estudio realizado a 100 pacientes con promedio de edad de 60 años en un Hospital de tercer nivel de Colombia, revelo una prevalencia de disfunción eréctil del $66 \%$ en su mayoría de grado leve. Relacionando sus causas, al hacer el análisis estadístico se encontró que la hipertensión fue la única causa que mostró significancia estadística, sugiriendo que es seis veces mas probable encontrarla en pacientes con disfunción eréctil ${ }^{9}$. En Honduras se realizó un estudio a 371 individuos en la ciudad de Siguatepeque, encontrándose que la frecuencia de disfunción eréctil fue de $58 \%$ (214), el grado de disfunción encontrado fue: leve 114 (53\%), de leve a moderada 57 (27\%), moderada 18 (8\%), y grave 25 $(12 \%)^{2}$; este estudio realizado en una ciudad a 120 kilómetros al norte de Tegucigalpa, tuvo un resultado idéntico a lo encontrado en el CAIAM; evidenciando que en la zona centro de Honduras la frecuencia de disfunción eréctil en hombres hipertensos es alta. Creemos que los datos de frecuencia de disfunción eréctil en hipertensos van en aumento paralelo a la hipertensión tal y como se ve en datos

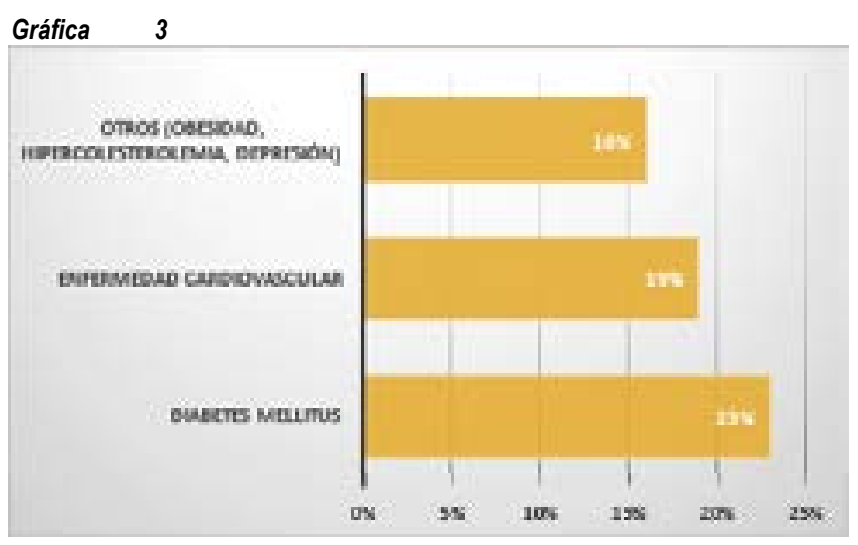

Tabla 2. Frecuencia de disfunción eréctil en hipertensos por rango de edad, Tegucigalpa 2013.

\begin{tabular}{|cc|}
\hline Edad & $\%(N)$ \\
\hline $60-70$ años & $15 \%(31)$ \\
\hline $70-80$ años & $24 \%(51)$ \\
\hline $80-90$ años & $19 \%(40)$ \\
\hline TOTAL & $58 \%(122)$ \\
\hline
\end{tabular}

brindados por la OMS en los que se observa que el número de personas afectadas aumentó de 600 millones en 1980 a 1000 millones en el 2008, cifra que hasta la fecha va en ascenso $^{10}$.

De igual manera en otro estudio donde su muestra fue de 100 pacientes en Colombia, se encontró $69 \%$ de hombres con disfunción eréctil ${ }^{11}$; un similar resultado se encontró en un estudio realizado en México, donde se tomó una muestra de 479 pacientes diabéticos encontrando $67 \%$ de ellos con disfunción eréctil ${ }^{12}$. Este aumento de casos con relación a este estudio realizado en Tegucigalpa puede deberse a una mayor prevalencia de hipertensión y diabetes en esos países con relación a Honduras ${ }^{13}$.

No obstante, en un estudio realizado en España en el que se estudiaron 2453 pacientes, se encontró que el $76 \%$ eran hipertensos; y de estos se encontró un $40 \%$ con algún grado de disfunción eréctil ${ }^{14}$. Otro estudio de este país con una muestra de 512 pacientes hipertensos reportó un $46.5 \%$ de hombres con cierto grado de disfunción eréctil. Se puede observar fluctuacion en los resultados de esos estudios con el nuestro, lo cual lo atribuimos a que la diferencia de la población con disfunción eréctil varia según el país ${ }^{15}$.

Un hallazgo de nuestro estudio así como en los otros estudios nacionales e internacionales, fue que en los pacientes masculinos mayores de 70 años, se encontró mayor cantidad de hombres con alteración de la función eréctil. Esto sugiere que el deterioro del endotelio vascular va incrementando junto con el proceso normal de envejecimiento ${ }^{7}$.

Respecto a la diabetes mellitus la prevalencia de disfun- 
ción eréctil es entre el 20 y el 50\%; su aparición suele ser de 10 a 15 años más temprano que en la población general ${ }^{7}$. La disfunción eréctil es un signo de enfermedad vascular en los sujetos con diabetes mellitus tipo $2^{14}$. En el mismo estudio realizado en Siguatepeque, Honduras, se encontró que del $58 \%$ de pacientes con disfunción eréctil el $31 \%$ fueron hipertensos con un $20 \%$ de diabéticos ${ }^{2}$. En el CAIAM, del $58 \%$ de pacientes hipertensos con disfunción eréctil, el $23 \%$ presentó además diabetes mellitus, evidenciándose porcentajes similares entre pacientes de ambas ciudades en el mismo país. A diferencia del estudio en México antes mencionado en el que se observo $67 \%$ de frecuencia de disfunción eréctil en paciente diabético y $72 \%$ de asociación de hipertensión arterial con diabetes mellitus ${ }^{12}$. Esto nos afirma que la diabetes mellitus también representa un alto factor de riesgo para padecer disfunción eréctil ${ }^{7}$.

La prevalencia encontrada en el CAIAM fue similar a la observada en otros estudios a mayor, menor e igual escala; comprobando que la disfunción eréctil es un problema a nivel mundial de alta prevalencia, la cual va en aumento. En nuestra población geriátrica hipertensa que asiste a la consulta externa del Centro de Atención Integral del Adulto
Mayor, se confirma esa alta prevalencia en hombres de edad avanzada, vulnerables a padecer enfermedades cardiovasculares que conlleven a la aparición de la disfunción eréctil. Esta conjetura deberá de confirmarse con la realización de estudios más profundos sobre la relación de las enfermedades cardiovasculares con la disfunción eréctil en Honduras.

\section{AGRADECIMIENTOS}

Agradecemos al Doctor Ángel Pineda, por facilitar la aplicación de instrumentos en el Centro de Atención Integral del Adulto Mayor (CAIAM) de la ciudad de Tegucigalpa.

\section{REFERENCIAS}

1. Romero C. Disfunción eréctil y enfermedad cardiovascular. Revista. Uruguay. Cardiología 2008; 23(1): 65- 70.

2. E. Wespes, E. Amar, I. Eardley, F. Giuliano, D. Hatzichristou, K. Hatzimouratidis, F. Montorsi, Y. Vardi DISFUNCIÓN SEXUAL MASCULINA: Disfunción eréctil y eyaculación precoz (Actualización del texto: Marzo de 2009) European Association of Urology 2010.

3. Roger S. Culley C. Goldstein I. Wyllie M. ERECTILE DYSFUNCTION: A CLINICAL GUIDE. Segunda Edición, USA, Informa HealthCare, New York 2009. P. 3.

4. Fauci A. Kasper D. Longo D. Loscalzo J. Jameson J. Hauser S. Braunwald E., Harrison Principios de Medicina Interna. Tomo 1. 17 edición McGraw-Hill interamericana, 2009. Pag.296-300

5. Odriozola A., Quintanilla M., Arias Pereira J., Tamayo A., Gonzales G., Disfunción Eréctil De Origen Vascular, Hospital de Galdako-Usansolo. Vizcaya, España, Arch Esp. Urol. Vol 63 No.8 Oct 2010

6. Rodriguez A., Roldan C., Marcos M., Rios R., Sarek I., Lopezo J.Factores Influyentes en la aparición de Disfunción Eréctil. JONNPR. 2016;1(3):107-114.

7. Zavala G., Núñez R., Portillo E., Reyes A., Bourdeth A., Sergio

D. DISFUNCIÓN ERÉCTIL EN POBLACIÓN MASCULINA MAYOR DE 35 AÑOS EN LA CIUDAD DE SIGUATEPEQUE (Erectile dysfunction in men above 35 in Siguatepeque, Honduras) Rev. Fac. Ciencias Médicas Enero-junio 2011 Vol 8(1) P.9-20 8. Castro R., Hernández P., Casilda R., García J., et al EPIDEMIOLOGÍA DE LA DISFUNCIÓN ERÉCTIL. FACTORES DE RIESGO, Unidad de Andrología Medicina Sexual y Reproductiva. Unidad Clínica de Urología. Hospital Regional Universitario Reina Sofía. Córdoba. España. Arch. Esp. Urol. 2010; 63 (8): 637-639.

9. Robayo A., Garcia P., Herneng A., Carbonell G., Prevalencia de Disfunción Eréctil y factores asociados en un hospital de tercer nivel, Universidad del Valle, Colombia; Urol. Colom. Vol XVIII, No.1:pp 39-46, 2009.

10. OMS, Información general sobre hipertensión en el mundo, Ginebra, Suiza 2013, http://apps.who.int/iris/ bitstream/10665/87679/1/WHO_DCO_WHD_2013.2_spa.pdf
11. Díaz C., Arias C., Barrios J., Ballestas C., Prevalencia de disfunción eréctil en pacientes hipertensos del Hospital Universitario del Caribe, Sociedad Colombiana de Urología Mayo 2013 Volumen 22 (1), P. 41-46.

12. Fong E., JaramilloA., Prevalencia de disfunción eréctil en pacientes diabéticos, México, Med Int., 2007, Vol.23 (6) P. 477-80. 13. OPS, fundación hondureña de diabetes, secretaria de salud. Iniciativa Centroamericana de diabetes (CAMDI). Encuesta de diabetes, hipertensión y factores de riesgo de enfermedades crónicas. Tegucigalpa, Honduras 2009.

14. Juanatey J., Ezquerra E., Barbera R., Salvador M., et al. .Disfuncion Eréctil Como Marcador De Vasculopatía En La Diabetes Mellitus En España, Elsevier Med Clin (Barc). 2009; 132(8):291-297.

15.. Cuéllar de León A., Campos González J., Muntó F., Martínez A., Gosalbes Soler V., Prosper Sierra M., Silvestre Quilez S., Valero García P., HIPERTENSIÓN ARTERIAL Y DISFUNCIÓN ERÉCTIL Revista de Urología de la SVMFYC España 2011. 